\title{
Alterations of microbiota in urine from women with interstitial cystitis
}

\author{
Huma Siddiqui ${ }^{1}$, Karin Lagesen ${ }^{1}$, Alexander J Nederbragt' ${ }^{1}$, Stig L Jeansson ${ }^{2}$ and Kjetill S Jakobsen ${ }^{1 *}$
}

\begin{abstract}
Background: Interstitial Cystitis (IC) is a chronic inflammatory condition of the bladder with unknown etiology. The aim of this study was to characterize the microbial community present in the urine from IC female patients by 454 high throughput sequencing of the $16 \mathrm{~S}$ variable regions V1V2 and V6. The taxonomical composition, richness and diversity of the $\mathrm{IC}$ microbiota were determined and compared to the microbial profile of asymptomatic healthy female (HF) urine.

Results: The composition and distribution of bacterial sequences differed between the urine microbiota of IC patients and HFs. Reduced sequence richness and diversity were found in IC patient urine, and a significant difference in the community structure of IC urine in relation to HF urine was observed. More than $90 \%$ of the IC sequence reads were identified as belonging to the bacterial genus Lactobacillus, a marked increase compared to $60 \%$ in HF urine.

Conclusion: The 165 rDNA sequence data demonstrates a shift in the composition of the bacterial community in IC urine. The reduced microbial diversity and richness is accompanied by a higher abundance of the bacterial genus Lactobacillus, compared to HF urine. This study demonstrates that high throughput sequencing analysis of urine microbiota in IC patients is a powerful tool towards a better understanding of this enigmatic disease.
\end{abstract}

\section{Background}

Interstitial Cystitis or Painful Bladder Syndrome (IC/ PBS) is a chronic condition characterized by frequent urination and bladder pain, which often results in reduced quality of life. Clinicians experience that this disease is becoming more prevalent [1]. While evidence suggests that about $90 \%$ of those affected are female, some urologists consider chronic bacterial prostatitis to be the male equivalent of IC.

The traditional definition of this illness is chronic sterile bladder inflammation of unknown etiology and it has not been possible to prove any causative pathogenic agent for this syndrome [2,3]. Currently there are four major hypotheses of pathogenesis: 1) autoimmunity, 2) deficiency of the glycosaminoglycan layer causing increased bladder wall permeability, 3) neurogenic inflammation and 4) chronic infection [4].

\footnotetext{
* Correspondence: kjetill.jakobsen@bio.uio.no

'Department of Biology, Centre for Ecological and Evolutionary Synthesis (CEES), University of Oslo, P.O. Box 1066, Blindern, 0316 Oslo, Norway Full list of author information is available at the end of the article
}

While several features of IC have suggested an infective etiology, numerous studies using traditional culture techniques have failed to provide consistent evidence that IC is associated with infection. It has been proposed that possible microbial agents causing this disease could be difficult to cultivate or are present in numbers too low to be confirmed in the laboratory [5]. Advances in molecular-based diagnostics have made it possible to overcome the limitations of culture-based detection. Investigators have used PCR, cloning and $16 \mathrm{~S}$ ribosomal DNA (rDNA) sequencing to search for pathogenic agents in bladder biopsies and urine specimens of IC patients [6-11], but with conflicting results. However, some of these studies have indicated that women with IC may have a higher prevalence of bacteria in the urine than those without IC $[6,8,9]$.

Furthermore, clinical studies have demonstrated that administration of antibiotics may sometimes be correlated with decreased symptoms in patients [12-14]. This can be due to both inhibition of bacterial growth or as a conventional anti-inflammatory effect of doxycycline. A study by Zhang et al. (2010) [15] not only demonstrates 
improvement in symptoms, but also a decreased level of nanobacteria after antibiotic treatment, strongly suggesting a microbial association of IC in some cases.

We recently developed approaches to assess the major microbial populations in female human urine, based on $16 \mathrm{~S}$ rDNA PCR followed by 454 pyrosequencing and analyses using a suite of bioinformatics tools (Siddiqui et al. (2011) [16]. We have shown that healthy female (HF) urine is a complex milieu with many different bacteria present. The normal human urine microbiota includes numerous fastidious and anaerobic microbes, which are potentially pathogenic [16-19]. In this work we applied these techniques in a prospective study to describe the microbial community present in urine from IC patients. We also performed a comparative analysis between the IC sequence dataset and the HF dataset previously generated [16] to determine to what extent the bacterial profiles differ. Our analyses indicate important differences between the two microbiota. We observe a lower complexity and variation between urine from IC individuals in relation to HF individuals.

\section{Methods}

\section{Urine sampling}

This study was approved by the Regional Committee for Medical Research Ethics East -Norway (REK Øst Prosjekt 110-08141c 1.2008.367), and the samples were taken with informed consent. 8 female patients of age from 27 to 67 years $(\mathrm{P} 1=59, \mathrm{P} 2=40, \mathrm{P} 3=27, \mathrm{P} 4=47, \mathrm{P} 5=31$, $\mathrm{P} 6=35, \mathrm{P} 7=32, \mathrm{P} 8=67)$ underwent thorough clinical examination including cystoscopy and fulfilled the criteria of European Society for the Study of Interstitial Cystitis (ESSIC) [20]. All patients had an established diagnosis of IC for more than four years. Midstream urine $(30 \mathrm{ml})$ was collected by the clean catch method with labial separation supervised by an urotherapy nurse. Specimens were kept at $4^{\circ} \mathrm{C}$, and within an hour processed for DNA isolation. All specimens used were culture-negative, as tested by the Urological Clinic at the University Hospital HF Aker-Oslo. None of the patients was receiving antibiotics at the time samples were taken, nor prior to that according to hospital records.

\section{Sample processing and DNA isolation}

Sample processing and DNA extraction was performed as previously described in Siddiqui et al. (2011) [16]. Briefly, urine aliquots $(30 \mathrm{ml})$ were pelleted by centrifugation and total DNA was isolated from sediments using DNeasy Blood \& Tissue kit (QIAGEN, Germany), preceded by incubation with POWERlyse (lysis buffer) (NorDiag ASA, Oslo, Norway). Finally, the DNA was eluted in $100 \mu \mathrm{l}$ of AE buffer from the kit. The DNA concentrations in the samples (P1-P8) were measured by Quant-iT
PicoGreeen dsDNA assay kit (Molecular Probes, Invitrogen USA) and ranged from $0.22 \mathrm{ng} / \mu \mathrm{l}$ to $4.36 \mathrm{ng} / \mu \mathrm{l}$.

\section{$16 \mathrm{~S}$ rDNA PCR and 454-pyrosequencing}

For each IC urine sample, we amplified 16S rDNA sequences using two different primer sets specific for the V1V2 and V6 hypervariable regions followed by 454 pyrosequencing as described in Siddiqui et al. (2011) [16]. Each of the primers consisted of a target specific region at their 3' end (V1V2 or V6) and an adapter sequence (Primer A or Primer B) at their 5' end as needed for GS FLX amplicon sequencing (454 Life Sciences, USA). Equal amounts of the two different amplicons (both V1V2- and V6-region) for a single subject were pooled and sequenced using GS-FLX chemistry in the same lane of a Pico-Titer plate divided into 16 lanes, except for samples P1, P2 and, P3, for which each amplicon (V1V2 and V6) was sequenced in a separate lane. 454 pyrosequencing was performed by the Norwegian Sequencing Centre (NSC) at the Department of Biology, University of Oslo, Norway.

\section{Sequence read preprocessing}

Sequence read preprocessing was done as described in Siddiqui et al. (2011) [16]. In brief, a total of 187,901 reads were produced from IC female urine samples. The initial sequence reads were split into two pools using the V1V2 and V6 primer sequences via the sfffile program from 454 Life Sciences (Roche), thus reducing the sequences to 172,931 IC urine reads (Table 1) due to the program splitting on an exact primer match. With a minimum length cutoff of 218 and 235 nt for the V1V2and V6-regions, respectively, the sequences were processed through the Pyronoise program [21] to reduce possible insertion/deletion errors at homopolymer runs. After denoising using Pyronoise, one sequence per cluster is retained together with the number of total reads mapping to that cluster.

The bacterial identification technique of broad range $16 \mathrm{~S}$ rDNA PCR is highly sensitive to environmental contamination. To control for this the IC urine sample sequence sets were stripped for sequences that could stem from contamination sources. This was done by using contamination control sequences (total $=25,246$ ) from negative control extractions (buffer and kit reagents) followed by PCR and pyrosequencing, as reported in Siddiqui et al. (2011) [16]. A complete linkage clustering at $1 \%$ genetic difference of each sample together with its respective control was performed using ESPRIT (http:// www.biotech.ufl.edu/people/sun/esprit.html [22]). Any sample sequences found in clusters where $\geq 50 \%$ of the sequences belonged to the contamination control were excluded from subsequent analyses (details in Siddiqui $e t$ al. (2011) [16]). 
Table 1 Sampling depth and biodiversity found by amplicon 454 pyrosequencing V1V2 and V6 region from urine

\begin{tabular}{|c|c|c|c|c|}
\hline & \multicolumn{2}{|c|}{ Combined sequence pool from HF urine ${ }^{1}$} & \multicolumn{2}{|c|}{ Combined sequence pool from IC urine ${ }^{2}$} \\
\hline & V1V2 & V6 & V1V2 & V6 \\
\hline \multicolumn{5}{|l|}{ Preprocessing } \\
\hline Total reads & 78346 & 74067 & 74211 & 98720 \\
\hline Length cutoff $^{3}$ & 48861 & 45382 & 46272 & 62325 \\
\hline Denoised $^{4}$ & 48860 & 45136 & 46267 & 62173 \\
\hline Cleaned $^{5}$ & 48452 & 44760 & 46138 & 62032 \\
\hline \multicolumn{5}{|l|}{ Taxonomy analysis } \\
\hline Phyla ${ }^{6}$ & 10 & 8 & 5 & 7 \\
\hline Genera $^{6}$ & 35 & 28 & 23 & 25 \\
\hline \multicolumn{5}{|l|}{ OTU and Diversity indices } \\
\hline Cleaned $^{5}$ & 48452 & 44760 & 46138 & 62032 \\
\hline Silva 165 alignment ${ }^{7}$ & 46001 & 44146 & 44594 & 61170 \\
\hline Unique OTUs & 974 & 2045 & 514 & 1432 \\
\hline OTUs $^{8}(3 \%)$ & 724 & 1537 & 344 & 1008 \\
\hline OTUs $^{8}(6 \%)$ & 615 & 1265 & 292 & 786 \\
\hline Chao $19^{9}(3 \%)$ & 1435 & 3936 & 357 & 2485 \\
\hline Chao1 LCl95 & 1261 & 3521 & 675 & 2172 \\
\hline Caho1 HCl95 & 1664 & 4437 & 1137 & 2883 \\
\hline Shannon index ${ }^{10}(3 \%)$ & 2.62 & 3.02 & 1.67 & 1.95 \\
\hline Inverse Simpson index ${ }^{11}(3 \%)$ & 6.97 & 7.03 & 3.57 & 3.72 \\
\hline \multicolumn{5}{|c|}{$\begin{array}{l}{ }^{1} \text { Combined sequence data from eight healthy female (HF) urine samples, sequences generated in prior study (Siddiqui et al. (2011) [16]). } \\
{ }^{2} \text { Combined sequence data from eight interstitial cystitis (IC) urine samples. } \\
\text { 3 } 2 \text { Length cutoff at minimum } 218 \mathrm{nt} \text { for V1V2 and } 235 \mathrm{nt} \text { for V6 reads. } \\
{ }^{4} \text { Total number of sequences after processing the dataset through Pyronoise [21]. } \\
\text { She number of reads per dataset after removal of sequences that could be from the same source as those in the contamination control dataset as described in } \\
\text { Siddiqui et al. (2011) [16]. } \\
{ }^{6} \text { Number of phyla and genera based on taxonomic classification by MEGAN V3.4 [23,24]. } \\
7 \text { The number of total reads after Silva } 16 \mathrm{~S} \text { alignment as recommended by MOTHUR [29]. } \\
{ }^{8} \text { OTUs: Operational Taxonomic Units at } 3 \% \text { or } 6 \% \text { nucleotide difference. } \\
{ }^{9} \text { Chao } 1 \text { is an estimator of the minimum richness and is based on the number of rare OTUs (singletons and doublets) within a sample. } \\
{ }^{10} \text { The Shannon index combines estimates of richness (total number of OTUs) and evenness (relative abundance). } \\
{ }^{11} \text { Inverse Simpson index (1/D) is an indication of the richness a community with uniform evenness would have at the same level of diversity. It takes into accoun } \\
\text { the number of OTUs present, as well as the abundance of each OTU. }\end{array}$} \\
\hline
\end{tabular}

Sequence data generated in this study were submitted to the Sequence Read Archive with the study accession number ERP001705. The dataset is available at http:// www.ebi.ac.uk/ena/data/view/ERP001705.

\section{Taxonomical analysis}

For taxonomic grouping of the sequence reads, MEGAN V3.4 http://www-ab.informatik.uni-tuebingen.de/software/ megan/welcome.html $[23,24]$ was used. First, the sequence reads were compared to a curated version of the SSUrdp database [25] using blastn with a maximum expectation value $(E)$ of $10^{-5}$. To reflect the actual abundance behind every denoised sequence cluster, each entry in the blast result file was replicated as many times as the total number of reads that mapped to that query sequence (for detailed procedure and parameters see Siddiqui et al. (2011) [16]). When comparing the individual datasets using MEGAN, numbers of reads were normalized up to 100,000 for every dataset.
Metastats, statistical methods (http://metastats.cbcb. umd.edu/, [26,27]) for detecting differentially abundant taxa, was used to reveal significant differences between IC urine microbiota and HF urine microbiota (taxonomy assessed in Siddiqui et al. 2011 [16]). This method employs a false discovery rate to improve specificity in high-complexity environments, and in addition handles sparsely sampled features using Fisher's exact test. The Metastats $p$ - values at different taxon levels, which were assigned using MEGAN, are listed in Additional file 1: Table S1. A $p$ - value $\leq 0.05$ was considered significant.

\section{Comparative OTU based clustering analysis of IC and HF urine}

Numbers of operational taxonomical units (OTUs), rarefaction curves and diversity indices were calculated using MOTHUR v1.22.2 [28,29] (see Table 1). To enable comparisons, the HF sequences generated in Siddiqui et al. (2011) [16] were reanalyzed along with 
the IC dataset from this study. Briefly, the sequences were aligned to the Silva $16 \mathrm{~S}$ alignment as recommended by MOTHUR [29] - sequences not aligned or aligned outside of where $95 \%$ of all of the sequences aligned were removed from the datasets. For an improved OTU clustering single linkage preclustering [30] was performed, allowing two nucleotides to differ between sequences, before clustering using average linkage. The processing was done both on each separate sample and on pooled V1V2 and V6 sequences for both IC and HF samples. We also calculated the OTUs and Shannon index for normalized numbers of sequences for each separate sample [31]. A random number of reads, corresponding to the lowest number of sequences in a sample group, i.e. 2,720 for V1V2 and 2,988 for V6, was picked 100 times from each sequence set. These new sequence sets were processed through MOTHUR in the same fashion as the full sequence sets and the average of the resulting OTUs and Shannon values are shown in Additional file 2: Table S2.

The differences in Shannon indices and inverse Simpson indices for the two communities (IC and HF urine) were also statistically evaluated by Wilcox rank sum test in R, for both the V1V2 and V6 amplicon datasets.

Venn diagrams were generated for both data sets using MOTHUR to calculate how many OTUs were shared between the two communities. To further explore the relationships between the two microbial communities, samples were clustered into Newick-formatted trees (using the UPGMA algorithm) with distance between communities calculated with $\theta \mathrm{YC}$ coefficient as a measurement of dissimilarity between community structures [32] in MOTHUR. In addition, weighted UniFrac testing [33] was performed to determine the statistical significance of clustering within the tree. A non-metric multidimensional scaling (NMDS) plot was generated in R for the distances calculated using $\theta \mathrm{YC}$ measures for each sequence dataset (V1V2 and V6), knowing that $\theta \mathrm{YC}$ weighs rare and abundant OTUs more evenly than other metrics such as Jaccard.

\section{Results}

\section{4 pyrosequenced $16 \mathrm{~S}$ rDNA amplicon sequences}

After preprocessing of the raw IC 454 reads as described in Siddiqui et al. (2011) [16], we obtained a total of 46, 138 and 62,032 16S rDNA sequences for V1V2 and V6 regions, respectively, see Table 1 . For comparison purposes, the preprocessing information for the HF urine sequences reported in Siddiqui et al. (2011) [16] is also listed in the table. Average number of reads per IC sample was 5,767 and 7,754 for V1V2 and V6, respectively (range: V1V2 3035-9506; V6 4900-14602) see Additional file 2: Table S2. 97\% of the preprocessed sequences were classified to phylum, order and family level, and $95 \%$ of the sequences were identified down to genus level.

\section{Composition of the IC urine microbiota}

In total, 7 phyla were identified by the $16 \mathrm{~S}$ rDNA sequences when the two different amplicon libraries (i.e. V1V2 and V6 $16 \mathrm{~S}$ regions) were considered together (Figure 1A). 93\% of the bacterial DNA sequences were assigned to Firmicutes, while the other $7 \%$ were assigned to 6 additional phyla. Actinobacteria was the second major phylum with $5 \%$ of the sequence abundance. Bacteroidetes and Tenericutes were represented by $1 \%$ of total bacterial sequences each, while three phyla - Proteobacteria, Fusobacteria and Nitrospirae - were detected by less than $1 \%$ of the assigned sequences.

In comparison to HF urine (Siddiqui et al. (2011) [16]), IC urine has a significantly higher proportion of Firmicutes $(p \leq 0.05, p$ value from Metastats for V1V2) (65\% vs $93 \%$, respectively) and reduced proportions of the other 5 common phyla (Figure 1A). Interestingly, the phylum Nitrospirae was only detected in IC urine. Five additional phyla present in HF urine (Siddiqui et al. (2011) [16]) were not identified in IC urine at all (Figure 1A). The distribution of major phyla in IC urine was similar for both the V1V2 and V6 sequence dataset, although Fusobacteria and Nitrospirae were only identified by the V6 sequence dataset.

Sequence reads for all phyla but one (Nitrospirae $0.003 \%$ of the reads) were further classified to order level. 16 of the 22 orders identified in healthy urine (Siddiqui et al. (2011) [16]) were also detected in IC urine. A significant shift in the bacterial composition was observed as a result of an increase of Lactobacillales (Figure $1 \mathrm{~B}$ and C) $(p \leq 0.05, p$ value from Metastats for V1V2) in the IC urine microbial community relative to HF urine. $92 \%$ and $91 \%$ of the reads for V1V2 and V6 respectively, were assigned to this order. In HF urine only $53 \%$ of the reads for V1V2 and 55\% for V6 were assigned to Lactobacillales. The abundance of other major orders seen in HF urine is reduced in IC samples (Figure 1B and Additional file 1: Table S1).

All sequence reads assigned to the order level were additionally assigned to family level. Among the 26 families identified, only 21 were assigned to different genera. Four of those families that were not further assigned (Pasteurelacae, Neisseriacae, Methyliphilaceae, and Micrococcaceae) were also detected in the HF urine study. Saprospiraceae, on the other hand was only found in IC urine.

At the genus level, the pooled sequences were assigned to 31 different genera, with 23 and 25 different genera for V1V2 and V6 analysis, respectively. Lactobacillus was the most abundant genus in both datasets and comprised a total of $92 \%$ of the sequences. Gardnerella and 


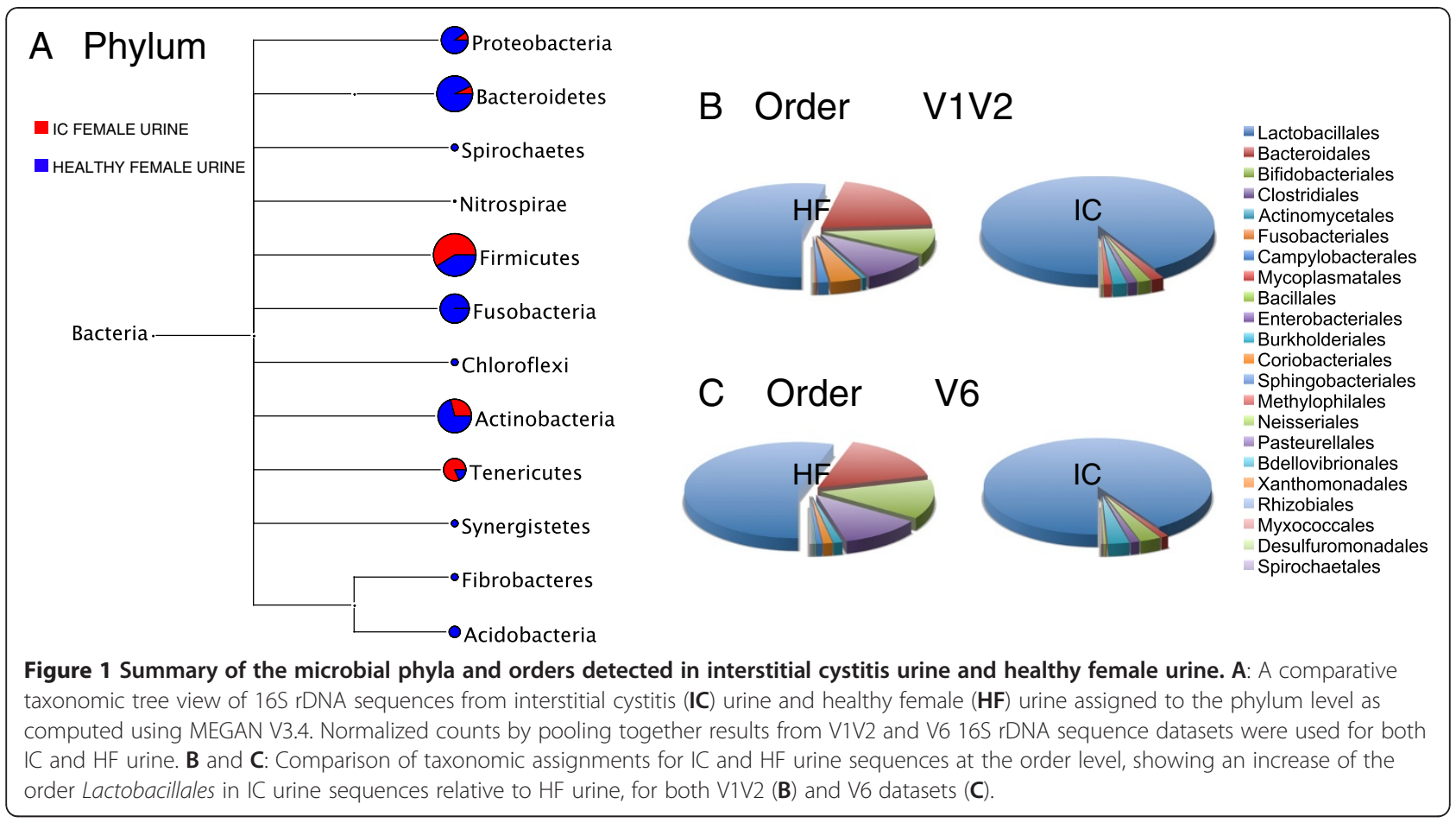

Corynebacterium were the two other major genera identified with $2 \%$ sequence abundance each. Prevotella and Ureaplasma were each represented by $1 \%$ of the sequences assigned. The other 26 genera determined in IC urine constituted only $2 \%$ of the total IC urine bacterial community.

In contrast to HF urine, there was a considerable reduction in total numbers of genera identified in IC urine (45 genera vs. 31 genera, respectively) (Additional file 1: Table S1). Additionally, the abundance of common genera was found to differ between IC patients and healthy females. The significant increase of Lactobacillus $(p \leq 0.05, p$ values from Metastats for both V1V2 and V6) in IC urine compared to HF urine again suggested a structural shift in the microbiota of IC patients. Enterococcus, Atopobium, Proteus and Cronobacter are 4 genera identified in IC urine that were not detected in our previous HF urine study, while a group of 17 genera were only associated with HF urine.

Sequences from 16 of the genera identified in the IC samples were further assigned to 22 different species (Additional file 3: Table S3). When comparing to our previous study, 13 of these species are already found in asymptomatic HF urine. However, nine of these species were not identified in our previous study, nor associated with IC according to literature.

\section{Variation between individual IC urine samples}

A clustering analysis using taxonomical data from both IC and HF individual urine samples is shown in Figure 2.
As previously demonstrated for HF urine (Siddiqui et al. 2011 [16]), variation between individuals was also evident for IC urine samples and a polymicrobial state was identified for all but one of the IC urine specimens. Although a clear clustering of samples from the two communities (IC and HF) was not apparent, we observed a narrower taxonomical range and reduced complexity in individual IC urine samples compared to the results from individual HF samples.

In all but two IC urine samples, Lactobacillus accounted for more than $\sim 95 \%$ of the sequences for both V1V2 and V6 data. Lactobacillus was not only the most abundant genus, but also the most frequent genus among all IC urine specimens with its rRNA sequences present in all eight samples, in contrast to urine samples from $\mathrm{HF}$ (6/8). Sequences assigned to Prevotella, Peptoniphilus and Anaerococcus were also frequently detected (5/8), followed by Staphylococcus and Finegoldia (4/8), and Gardnerella, Streptococcus and Dialister (3/8) in IC urine. Including Ureaplasma, 7 genera were identified by reads belonging to 2 urine samples and another 15 genera were only detected in 1 out of the 8 samples.

\section{Species richness and diversity}

Estimation of species richness and diversity were calculated for the two combined V1V2 and V6 sequence pools (Table 1), as well as for single urine samples (Additional file 2: Table S2). At the species level, defined as OTUs at $3 \%$ genetic difference, 344 species for the V1V2 and 1,008 species for the V6 sequence datasets were estimated in 


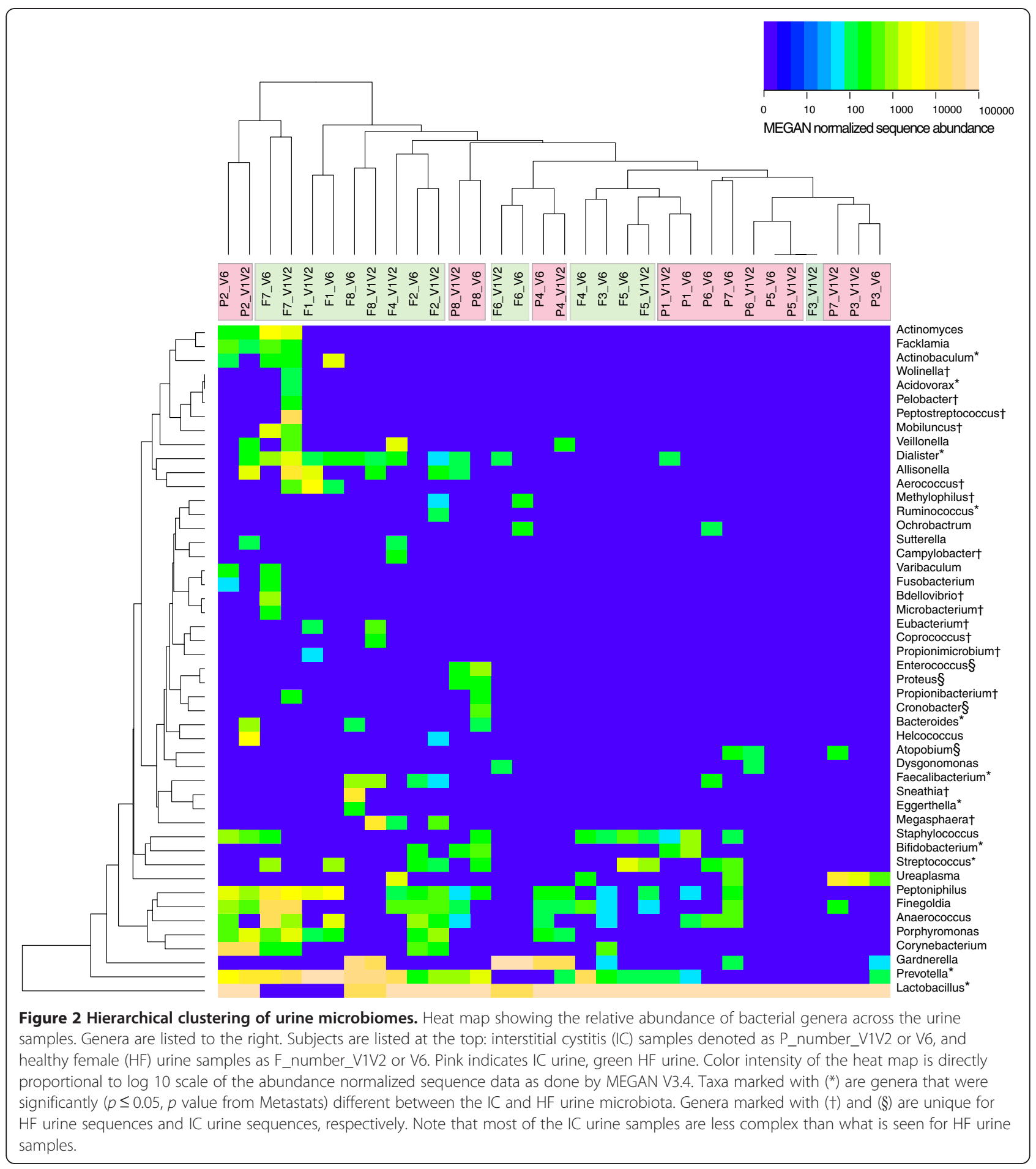

the IC urine community. At a more conservative level, defined as OTUs at 6\% dissimilarity level, 292 and 786 OTUs were estimated for V1V2 and V6, respectively. These numbers for richness are considerably lower than found in HF urine (Table 1 and Figure 3A). The number of OTUs at $3 \%$ difference for the individual samples for both IC and HF are indicated in box plots (Figure 3B) for both V1V2 and V6 analysis. In general, fewer number of OTU clusters were observed for IC individuals than that for HF individuals. Ecological diversity measured by Shannon and inverse Simpson indices also indicate lower diversity in IC urine in comparison to what was seen in urine from HF (Figure 3C and D). Specifically, a significant $(p<0.05)$ decrease in inverse Simpson index in IC 


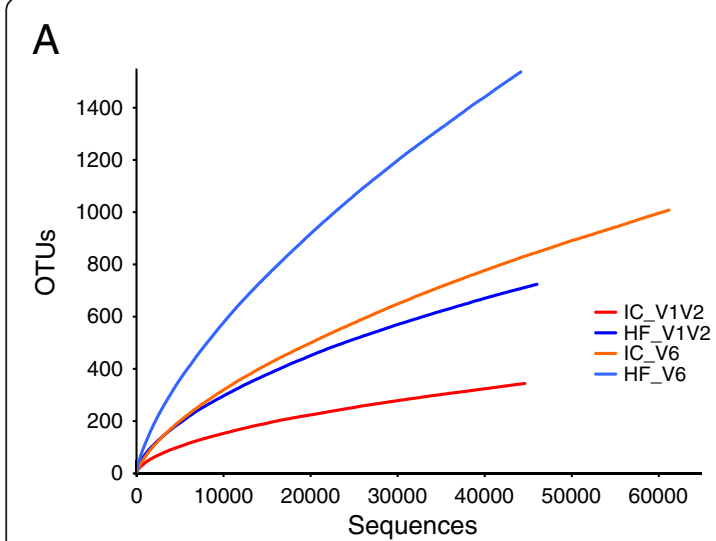

C

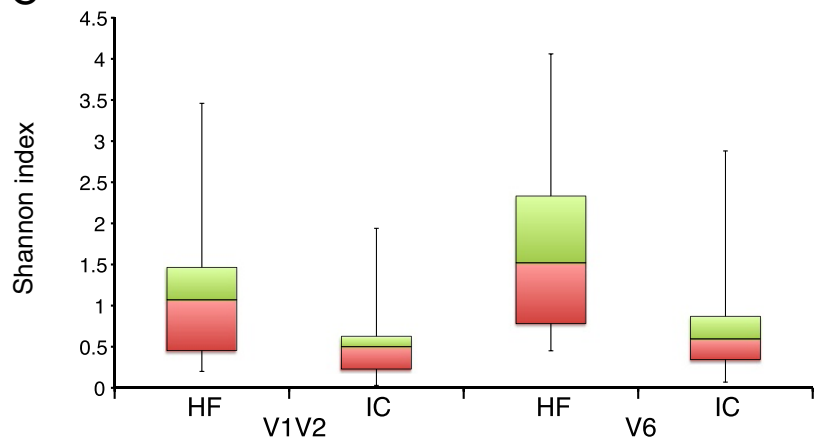

B

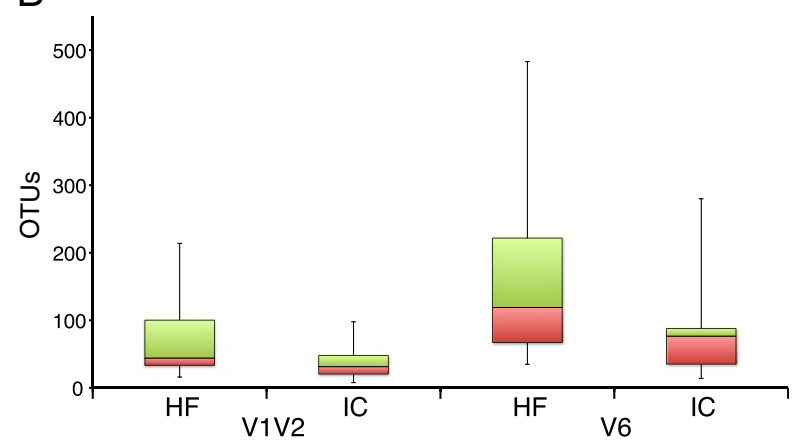

D

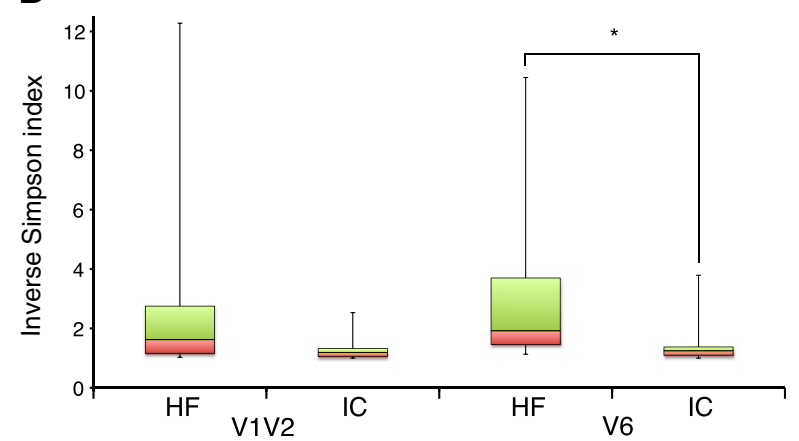

Figure 3 Comparison of richness and diversity estimations of urine from interstitial cystitis (IC) patients and healthy females (HF). A: Rarefaction curves depicting number of OTUs (at 3\% genetic difference) as function of the total number of sequences for the combined sequence pool datasets for IC urine V1V2 and V6 (red and orange) and HF urine V1V2 and V6 (dark and light blue). The curves show a decreased estimate of species richness in the IC urine microbiome compared to the HF urine microbiome. B, C, and D: Box plots showing richness and diversity of $16 \mathrm{~S}$ rDNA sequences. Boxes contain $50 \%$ of the data and have lines at the lower quartile (red), median and upper quartile (green) values. Ends of the whiskers mark the lowest and highest value. The plots show the results of a combined assessment of the eight urine samples in each HF and IC microbiome and with normalized numbers of sequences for OTU and Shannon index values (B and C). B: Observed OTU counts (at 3\% genetic difference) of all urine samples taken from HF and IC, for both V1V2 and V6 datasets. C and $\mathbf{D}$ : Shannon index and inverse Simpson index at $3 \%$ sequence dissimilarity calculated to estimate diversity for both V1V2 and V6 datasets. Asterisks $\left(^{*}\right)$ indicate significant differences (Wilcox rank sum test: * $p<0.05$ ). Note that a single sample (P2) in the IC community is the only outlier with the highest values for both richness and diversity (for both V1V2 and V6 analysis).

patients compared to HF was found for the V6 analysis. Taken together, the results for both V1V2 and V6 support each other and confirm that the urine community is less diverse in IC patients than in HF individuals. However, the single IC outlier with high richness and diversity (Figure 3B-D) also clustered outside the IC group in the clustering analysis done using taxonomy data (Figure 2) showing that there is also potential for variation within the IC community.

The IC and HF urine also showed a degree of community similarity at $3 \%$ sequence dissimilarity level - about $12 \%$ and $9.5 \%$ of the total OTUs for V1V2 and V6, respectively, were present in both groups (Additional file 4: Figure S1). To further explore the relationships between the two communities, $\theta \mathrm{YC}$ distances, taking into account both community association and relative abundance, were calculated at 3\% dissimilarity level for all samples. These distances for both V1V2 and V6 datasets were then visualized by NMDS plots; see Figure 4A and $\mathrm{B}$. Although an overlap between the two communities is detected, HF urine samples were more dispersed than IC samples. A pattern of less variation between samples from IC patients than for HF samples was suggested. Weighted UniFrac hypothesis testing for $\theta \mathrm{YC}$ distances confirmed the significance $(p<0.001)$ of the differences observed in the community structure.

\section{Discussion}

We have characterized the urine microbiota of IC patients using high throughput 454 pyrosesequencing of $16 \mathrm{~S}$ rDNA amplicons. These results were compared to HF data from our previous study (Siddiqui et al. (2011) [16]). 

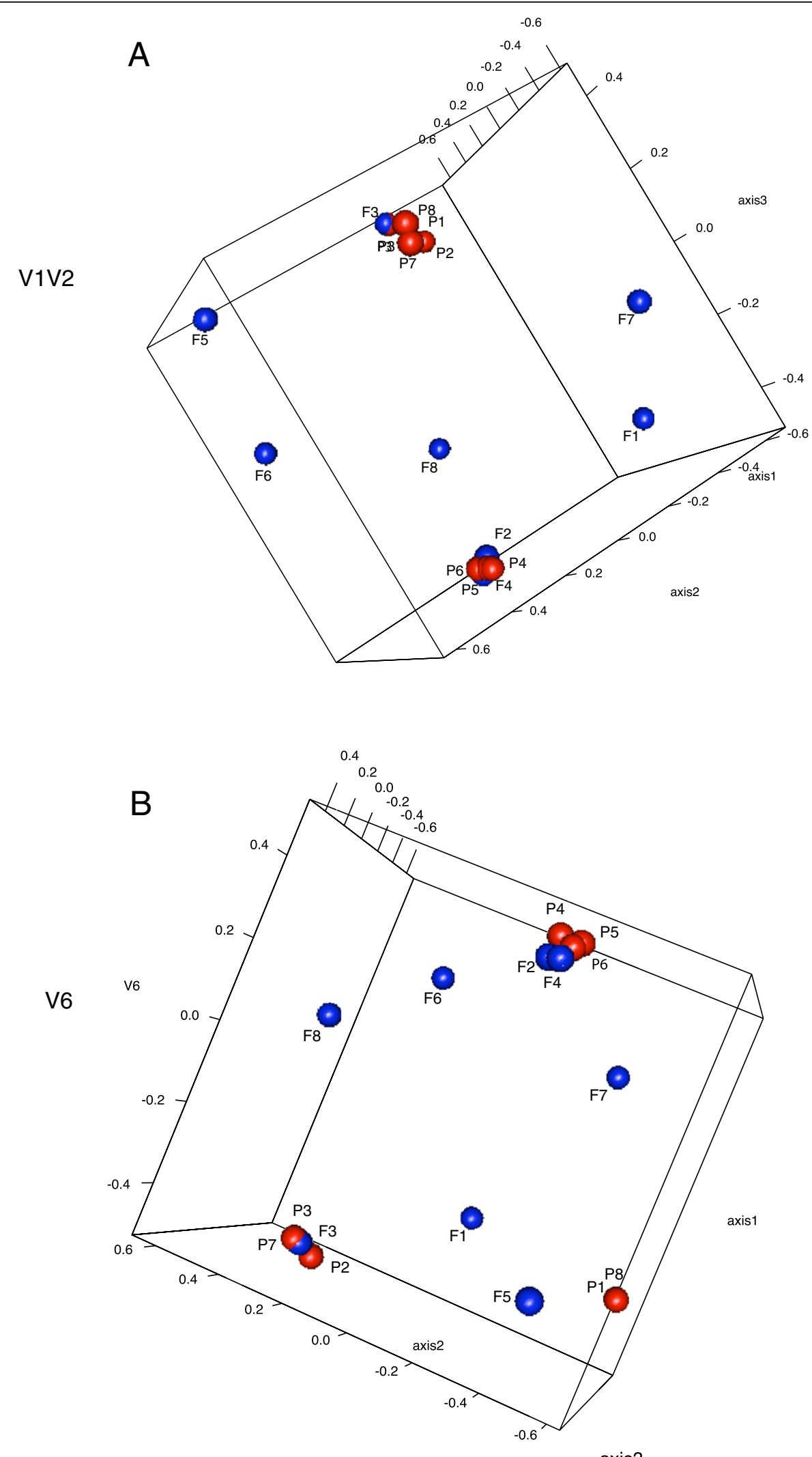

Figure 4 OTU based clustering analysis of urine microbiomes. Non-metric multidimensional scaling (NMDS) plots were generated based on $\theta \mathrm{YC}$ distances (0.03) between interstitial cystitis (IC) and healthy female (HF) microbiomes for both V1V2 (A) and V6 region (B). Red: IC patient samples; blue: HF samples. 
Our results did not reveal any single potential pathogenic bacterium common to all IC patients. However, important differences were detected between the IC and HF microbiota.

The use of primers for both V1V2 and V6 regions yielded complementary results for IC urine in line with the previous study of HF urine (Siddiqui et al. (2011) [16]), and thus maximized the detection of bacterial diversity.

Knowing that urine samples are at risk of contamination by bacterial flora of the female urogential system $[34,35]$, mid-stream urine sampling was performed under guidance of an experienced urotherapy nurse. Suprapubic puncture was suggested as an alternative method, but the method was considered to be too invasive. Interestingly, comparing results from our previous microbiome study on female mid-stream urine (Siddiqui et al. 2011) [16] with recent results from suprapubic aspirate by Wolfe et al. (2012) [19], the major findings are the same; a strong indication that mid-stream urine will give comparable results in a urine microbiome analysis.

\section{A decrease in species richness in IC urine}

A decrease in overall richness and ecological diversity (as indicated by rarefaction analysis, number of OTUs, Shannon index and inverse Simpson index estimations) of IC urine microbiota was detected in contrast to HF urine (Table 1 and Figure 3). In addition, the $\beta$-diversity analysis ( $\theta$ YC distances between all urine samples) suggested that the microbiota of HF samples are more dissimilar from each other than the microbiota of IC individuals.

The taxonomical analysis indicated a shift in composition of urine microbiota of IC patients, with changes in bacterial groups spanning from genus to phyla level and a reduction in microbial complexity compared to HF. More importantly, a significant increase in Lactobacillus in IC patients was revealed. Lactobacillus was detected in every IC urine sample and a total sequence abundance of $92 \%$ in contrast to $57 \%$ in HF urine was observed. This shift was also clearly displayed both at the order and phylum level (Lactobacillales and Firmicutes, respectively). In contrast, Prevotella, - a genus belonging to the phylum Bacteroidetes (order Bacteriodales) - was present only at $1 \%$, significantly lower than in HF urine, where it was previously reported as one of the major genera with an abundance of 19\%. Gardnerella, another dominant genus in female urine, was present with the same frequency in IC urine but with a general lower abundance.

A reduction in bacterial diversity and shift in the microbiota as observed in this chronic inflammatory state has also been reported for other clinical conditions such as obesity, irritable bowel syndrome, and inflammatory bowel disease including Crohn's disease [36-38].

\section{Bacteria associated with IC}

Attempts to identify an infectious etiology for IC have not yet found any evidence for a specific pathogen. However, previous culture-dependent studies of samples from IC patients (i.e. bladder biopsy, midstream urine) have reported organisms such as Gardnerella, Lactobacillus sp., Streptococcus ssp., Escherichia coli, Proteus mirabilis, Corynebacterium ssp., Klebsiella sp., Enterococcus sp., Propionbacterium, Prevotella, Bacteroides sp., and Peptostreptococcus [6,9,39]. Lactobacillus, Gardnerella and Streptococcus were repeatedly detected in these studies and were also seen in our study. Haarala et al. (1999) [9] using culture techniques concluded that bacterial flora of midstream urine from patients with IC clearly differs from that of healthy women, in line with our findings. A study by Zhang et al. (2010) [15] suggested nanobacteria as a possible causative agent for IC. The two latter studies also reported a reduction in bacterial levels and urinary symptoms upon antibiotic treatment of the IC patients. The primer pairs both for V1V2 and V6 amplicons used in our study would be expected to amplify $16 \mathrm{~S} \mathrm{rDNA}$ regions of all of the organisms mentioned above. Nevertheless we did not identify Klebsiella, E.coli, Peptostreptococcus or nanobacteria in any of our IC urine samples.

Studies reporting results from culture-independent $16 \mathrm{~S}$ rDNA PCR approaches on samples (i.e. bladder biopsy, midstream urine) from IC patients, have yielded somewhat conflicting results both in terms of positive PCRs and the resulting bacterial profiles $[7,8,10,11,40]$. While two of the reports $[11,40]$ found no evidence of bacterial DNA in biopsy and urine specimens from IC patients, Dominique et al. (1995) [8] demonstrated bacterial DNA in bladder tissues in 29\% of patients with IC. The 4 sequences retrieved showed homology to $E$. coli (2) and Pseudomonas (2), however neither of these bacteria was found in our study. Heritz et al. (1997) [10] also reported bacterial DNA in both biopsies and urines from IC patients (53\% and 46\%, respectively). They concluded that there is a difference in the bacterial profile between the patients and controls, and further suggested a link between one or more bacterial species and IC. However, in their study only 11 bacterial clones from 3 different IC patients were analyzed and the bacterial sequences were related to E.coli, Abiotrophia defectivus, Veillonella and Rothia dentocariosa. Except for Veillonella, these bacteria were not detected in our study. All these 4 previously reported studies used different primer sets (likely to explain some of the differences in the results) and classical cloning strategies (explaining the very few sequences analyzed). In contrast, our study represents the first $16 \mathrm{~S}$ rDNA amplicon high throughput sequencing approach on IC urine, increasing both the sensitivity and resolution of the investigation. 


\section{Significance of Lactobacillus in IC urine}

Lactobacillus has not only been indicated or shown in IC urine samples from females $(100 \%$ of the cases in this study and as shown by others $[6,9,39])$ but also demonstrated in IC urine from a male subject [41]. In our study we also detected a significant increase in abundance of this genus, considering its supposedly commensal presence in human urine from healthy subjects $[16,18,19]$.

Lactobacillus is generally considered to be of low virulence, rarely causing infections in humans. It is best known for its presence in vaginal microflora, where it normally generates and maintains a physiological acidic environment, which prevents infections. Because of these properties, Lactobacillus has been used in probiotics, and is thought to prevent or even treat urinary tract infection (UTI) [42]. However, there are increasing indications that specific Lactobacillus spp are of pathogenic relevance and may be involved in urinary tract infections $[43,44]$.

Many female patients with symptoms suggestive of UTI, but with culture-negative urines are often treated with antibacterial agents since their symptoms may be indistinguishable from those with a proven UTI [45]. It has been proposed that Lactobacillus, resistant to widely used antibiotics, may multiply during treatment, giving the genus an advantage over antibiotic-sensitive commensals, and allowing it to invade the proximal urethra and paraurethral tissues causing inflammatory changes [45]. This organism has also been related to the presence of UTI symptoms in otherwise culture-negative urines $[43,44,46]$. In a study by Maskell et al. (1983) [46] antibacterial treatment was withheld over the course of 2 years from symptomatic women with culture-negative urine. During the course of the study Lactobacilli (detected by special culture techniques) gradually disappeared from the urine of most of the patients who also became symptom free. A similar association of Lactobacillus and urinary symptoms was reported by Darbro et al. (2009) [44]. Over a six-month period a female patient had constant symptoms and high counts of Lactobacillus spp (> 50,000 CFU/ml) in the urine; she became symptomfree with culture-negative urine after treatment with Lactobacillus-targeted medication. These results also suggest that a shift in the microbial community towards Lactobacillus in IC urine samples may be an important etiological factor for the severe symptoms reported by the patients. Since additional culture techniques such as $48 \mathrm{~h}$ incubation in an atmosphere containing $7 \% \mathrm{CO}_{2}$ are needed for detection of Lactobacillus, this may be the reason why IC urine samples have not yet been associated with bacterial growth in routine clinical investigations. However, in our study this problem was overcome by a culture-independent approach.

\section{Conclusion}

This investigation did not reveal any obvious putative causative bacterial agents of IC. However, the greater abundance of Lactobacillus in IC urine and its lower occurrence in HF urine is an important finding that requires further study to establish whether these microbial changes play a part in the development of IC. To this end, whole genome sequencing of Lactobacillus from IC patients may be a possible approach. Even if an increased presence of Lactobacillus is merely a secondary marker, understanding its IC associated genomics could aid in diagnosis and therapeutic assessment.

\section{Additional files}

Additional file 1: Table S1. Differentially abundant taxa between interstitial cystitis (IC) and healthy female (HF) urine microbiota as estimated by Metastats (http://metastats.cbcb.umd.edu/).

Additional file 2: Table S2. Sampling depth and biodiversity found by amplicon 454 pyrosequencing V1V2 and V6 region from eight interstitial cystitis (IC) and eight healthy female (HF) urine.

Additional file 3: Table S3. Bacterial species identified in interstitial cystitis (IC) urine by $16 \mathrm{~S}$ rDNA amplicon 454 pyrosequencing.

Additional file 4: Figure S1. Venn diagrams for overlap between healthy female (HF) urine observed OTUs vs. interstitial cystitis (IC) urine OTUs, for both V1V2 (A) and V6 (B) region. The OTUs are calculated at 3\% genetic sequence dissimilarity.

\section{Authors' contribution}

HS, AJN, SLJ and KSJ were involved in study design; HS processed the samples and carried out the molecular techniques. KL and HS performed the bioinformatics and taxonomic analysis. HS interpreted the data and authored the manuscript. All authors edited and commented on the paper and all authors read and approved the final manuscript.

\section{Acknowledgements}

The authors would like to thank Hege Junita Gaup for technical assistance, William Ryan Easterday for critical reading of the manuscript and the Norwegian Sequencing Centre (NSC, www.sequencing.uio.no), Department of Biology, University of Oslo, for sequencing services. We are very grateful to Professor Lars M Eri and urotherapists Turid H Hoel and Bodil Svendsen at Aker University Hospital HF, Urological Clinic for specimen collection. Additionally we thank two anonymous reviewers, whose comments helped to improve the manuscript.

Financial support for this research was provided by grants from the Research Council of Norway to KSJ and from CEES to HS.

\section{Author details}

${ }^{1}$ Department of Biology, Centre for Ecological and Evolutionary Synthesis (CEES), University of Oslo, P.O. Box 1066, Blindern, 0316 Oslo, Norway. ${ }^{2}$ University Hospital HF Aker-Oslo and Faculty of Medicine, Division of Medicine, ME/CFS-center, University of Oslo, P.O. Box 4956, Nydalen, 0424 Oslo, Norway.

Received: 25 June 2012 Accepted: 5 September 2012

Published: 13 September 2012

\section{References}

1. Payne CK, Joyce GF, Wise M, Clemens JQ: Interstitial cystitis and painful bladder syndrome. J Urol 2007, 177(6):2042-2049.

2. Abrams P, Cardozo L, Fall M, Griffiths D, Rosier P, Ulmsten U, van Kerrebroeck $P$, Victor $A$, Wein A: The standardisation of terminology of lower urinary tract function: report from the Standardisation Subcommittee of the International Continence Society. Neurourol Urodyn 2002, 21(2):167-178. 
3. Marinkovic SP, Moldwin R, Gillen LM, Stanton SL: The management of interstitial cystitis or painful bladder syndrome in women. BMJ 2009, 339:b2707.

4. Bouchelouche K, Nordling J: Recent developments in the management of interstitial cystitis. Curr Opin Urol 2003, 13(4):309-313.

5. Hanno PM: Diagnosis of interstitial cystitis. Urol Clin North Am 1994, 21(1):63-66

6. Keay S, Schwalbe RS, Trifillis AL, Lovchik JC, Jacobs S, Warren JW: A prospective study of microorganisms in urine and bladder biopsies from interstitial cystitis patients and controls. Urology 1995, 45(2):223-229.

7. Keay S, Zhang CO, Baldwin BR, Jacobs SC, Warren JW: Polymerase chain reaction amplification of bacterial 16S rRNA genes in interstitial cystitis and control patient bladder biopsies. J Urol 1998, 159(1):280-283.

8. Domingue GJ, Ghoniem GM, Bost KL, Fermin C, Human LG: Dormant microbes in interstitial cystitis. J Urol 1995, 153(4):1321-1326.

9. Haarala M, Kiilholma P, Lehtonen OP: Urinary bacterial flora of women with urethral syndrome and interstitial cystitis. Gynecol Obstet Invest 1999, 47(1):42-44.

10. Heritz DM, Lacroix JM, Batra SD, Jarvi KA, Beheshti B, Mittelman MW: Detection of eubacteria in interstitial cystitis by $16 \mathrm{~S}$ rDNA amplification. J Urol 1997, 158(6):2291-2295.

11. Al-Hadithi HN, Williams H, Hart CA, Frazer M, Adams EJ, Richmond DH, Tincello DG: Absence of bacterial and viral DNA in bladder biopsies from patients with interstitial cystitis/chronic pelvic pain syndrome. J Urol 2005, 174(1):151-154.

12. Warren JW, Brown V, Jacobs S, Horne L, Langenberg P, Greenberg P: Urinary tract infection and inflammation at onset of interstitial cystitis/ painful bladder syndrome. Urology 2008, 71(6):1085-1090.

13. Burkhard FC, Blick N, Hochreiter WW, Studer UE: Urinary urgency and frequency, and chronic urethral and/or pelvic pain in females. Can doxycycline help? J Urol 2004, 172(1):232-235.

14. Smith SD, Wheeler MA, Foster HE Jr, Weiss RM: Improvement in interstitial cystitis symptom scores during treatment with oral L-arginine. J Urol 1997, 158(3 Pt 1):703-708.

15. Zhang QH, Shen XC, Zhou ZS, Chen ZW, Lu GS, Song B: Decreased nanobacteria levels and symptoms of nanobacteria-associated interstitial cystitis/painful bladder syndrome after tetracycline treatment. Int Urogynecol J Pelvic Floor Dysfunct 2010, 21(1):103-109.

16. Siddiqui $H$, Nederbragt AJ, Lagesen $K$, Jeansson SL, Jakobsen KS: Assessing diversity of the female urine microbiota by high throughput sequencing of 16S rDNA amplicons. BMC Microbiol 2011, 11:244.

17. Nelson DE, Van Der Pol B, Dong Q, Revanna KV, Fan B, Easwaran S, Sodergren E, Weinstock GM, Diao L, Fortenberry JD: Characteristic male urine microbiomes associate with asymptomatic sexually transmitted infection. PLOS One 2010, 5(11):e14116.

18. Dong Q, Nelson DE, Toh E, Diao L, Gao X, Fortenberry JD, Van Der Pol B: The microbial communities in male first catch urine are highly similar to those in paired urethral swab specimens. PLoS One 2011, 6(5):e19709.

19. Wolfe AJ, Toh E, Shibata N, Rong R, Kenton K, Fitzgerald M, Mueller ER, Schreckenberger P, Dong Q, Nelson DE, et al: Evidence of uncultivated bacteria in the adult female bladder. J Clin Microbiol 2012, 50(4):1376-1383.

20. van de Merwe JP, Nordling J, Bouchelouche P, Bouchelouche K, Cervigni M, Daha LK, Elneil S, Fall M, Hohlbrugger G, Irwin P, et al: Diagnostic criteria, classification, and nomenclature for painful bladder syndrome/interstitial cystitis: an ESSIC proposal. Eur Urol 2008, 53(1):60-67.

21. Quince C, Lanzen A, Curtis TP, Davenport RJ, Hall N, Head IM, Read LF, Sloan WT: Accurate determination of microbial diversity from 454 pyrosequencing data. Nat Methods 2009, 6(9):639-641.

22. ESPRIT. http://www.biotech.ufl.edu/people/sun/esprit.html.

23. MEtaGenome ANalyzer. http://www-ab.informatik.uni-tuebingen.de/software/ megan/welcome.html.

24. Huson DH, Auch AF, Qi J, Schuster SC: MEGAN analysis of metagenomic data. Genome Res 2007, 17(3):377-386. Software freely available for academic purposes from www-ab.informatik.uni-tuebingen.de/software/ megan.

25. Urich T, Lanzen A, Qi J, Huson DH, Schleper C, Schuster SC: Simultaneous assessment of soil microbial community structure and function through analysis of the meta-transcriptome. PLoS One 2008, 3(6):e2527.

26. Metastats. http://metastats.cbcb.umd.edu/.
27. White JR, Nagarajan N, Pop M: Statistical methods for detecting differentially abundant features in clinical metagenomic samples. PLOS Comput Biol 2009, 5(4):e1000352.

28. Schloss PD, Westcott SL, Ryabin T, Hall JR, Hartmann M, Hollister EB, Lesniewski RA, Oakley BB, Parks DH, Robinson CJ, et al: Introducing mothur: open-source, platform-independent, community-supported software for describing and comparing microbial communities. App/ Environ Microbiol 2009, 75(23):7537-7541.

29. Schloss PD, Gevers D, Westcott SL: Reducing the effects of PCR amplification and sequencing artifacts on $16 \mathrm{~S}$ rRNA-based studies. PLOS One 2011, 6(12):e27310

30. Huse SM, Welch DM, Morrison HG, Sogin ML: Ironing out the wrinkles in the rare biosphere through improved OTU clustering. Environ Microbiol 2010, 12(7):1889-1898.

31. Lemos LN, Fulthorpe RR, Triplett EW, Roesch LF: Rethinking microbial diversity analysis in the high throughput sequencing era. J Microbiol Methods 2011, 86(1):42-51.

32. Yue JC, Clayton MK: A similarity measure based on species proportions. Commun Stat Theor M 2005, 34(11):2123-2131.

33. Lozupone CA, Hamady M, Kelley ST, Knight R: Quantitative and qualitative beta diversity measures lead to different insights into factors that structure microbial communities. Appl Environ Microbiol 2007, 73(5):1576-1585.

34. Jackson SR, Dryden M, Gillett P, Kearney P, Weatherall R: A novel midstream urine-collection device reduces contamination rates in urine cultures amongst women. BJU Int 2005, 96(3):360-364.

35. Bekeris $L G$, Jones $B A$, Walsh MK, Wagar EA: Urine culture contamination: a College of American Pathologists Q-Probes study of 127 laboratories. Arch Pathol Lab Med 2008, 132(6):913-917.

36. Ott SJ, Musfeldt M, Wenderoth DF, Hampe J, Brant O, Folsch UR, Timmis KN, Schreiber S: Reduction in diversity of the colonic mucosa associated bacterial microflora in patients with active inflammatory bowel disease. Gut 2004, 53(5):685-693.

37. Carroll IM, Ringel-Kulka T, Siddle JP, Ringel Y: Alterations in composition and diversity of the intestinal microbiota in patients with diarrhea-predominant irritable bowel syndrome. Neurogastroenterol Motil 2012, 24(6):521-e248.

38. Turnbaugh PJ, Hamady M, Yatsunenko T, Cantarel BL, Duncan A, Ley RE, Sogin ML, Jones WJ, Roe BA, Affourtit JP, et al: A core gut microbiome in obese and lean twins. Nature 2009, 457(7228):480-484.

39. Wilkins EG, Payne SR, Pead PJ, Moss ST, Maskell RM: Interstitial cystitis and the urethral syndrome: a possible answer. Br J Urol 1989, 64(1):39-44.

40. Haarala M, Jalava J, Laato M, Kiilholma P, Nurmi M, Alanen A: Absence of bacterial DNA in the bladder of patients with interstitial cystitis. J Urol 1996, 156(5):1843-1845.

41. Lacroix JM, Jarvic K, Batrab SD, Heritze DM, Mittelman MW: PCR-based technique for the detection of bacteria in semen and urine. J Microbiol Methods 1996, 26(1-2):61-71.

42. Falagas ME, Betsi Gl, Tokas T, Athanasiou S: Probiotics for prevention of recurrent urinary tract infections in women: a review of the evidence from microbiological and clinical studies. Drugs 2006, 66(9):1253-1261.

43. Imirzalioglu C, Hain T, Chakraborty T, Domann E: Hidden pathogens uncovered: metagenomic analysis of urinary tract infections. Andrologia 2008, 40(2):66-71.

44. Darbro BW, Petroelje BK, Doern GV: Lactobacillus delbrueckii as the cause of urinary tract infection. J Clin Microbio/ 2009, 47(1):275-277.

45. Maskell RM: The natural history of urinary tract infection in women. Med Hypotheses 2010, 74(5):802-806.

46. Maskell R, Pead L, Sanderson RA: Fastidious bacteria and the urethral syndrome: a 2-year clinical and bacteriological study of 51 women. Lancet 1983, 2(8362):1277-1280.

doi:10.1186/1471-2180-12-205

Cite this article as: Siddiqui et al.: Alterations of microbiota in urine from women with interstitial cystitis. BMC Microbiology 2012 12:205. 